\title{
Investigating Antimicrobial Peptide-Membrane Interactions Using Fast Photochemical Oxidation of Peptides in Nanodiscs
}

\author{
Deseree J. Reid¹, James G. Rohrbough¹, Marius M. Kostelic ${ }^{1}$, and Michael T. Marty1,2,* \\ ${ }^{1}$ Department of Chemistry and Biochemistry and ${ }^{2}$ Bio5 Institute, University of Arizona, Tucson, AZ 85721, USA
}

\begin{abstract}
Antimicrobial peptides (AMPs) are an important part of the innate immune system and demonstrate promising applications in the fight against antibiotic resistant infections due to their unique mechanism of targeting bacterial membranes. However, it is challenging to study the interactions of these peptides within lipid bilayers, making it difficult to understand their mechanisms of toxicity and selectivity. Here, we used fast photochemical oxidation of peptides, an irreversible footprinting technique that labels solvent accessible residues, and native charge detection-mass spectrometry to study AMP-lipid interactions with different lipid bilayer nanodiscs. We observed differences in the oxidation of two peptides, indolicidin and LL-37, in three distinct lipid environments, which reveals their affinity for lipid bilayers. Our findings suggest that indolicidin interacts with lipid head groups via a simple charge-driven mechanism, but LL-37 is more specific for E. coli nanodiscs. These results provide complementary information on the potential modes of action and lipid selectivity of AMPs.
\end{abstract}

\section{INTRODUCTION}

Antimicrobial peptides (AMPs) are mainly cationic, amphipathic peptides that target bacterial membranes, disrupting their integrity and destroying the bacterial cell. ${ }^{1}$ An important part of the innate immune system in animals, they are also found in plants, fungi, and prokaryotes. ${ }^{2}$ Rather than targeting specific proteins, AMPs directly target cell membranes, making them potential therapeutics for antibiotic resistant bacteria. ${ }^{3}$ However, AMP therapies have been held back by the poor understanding of their mechanism of action and their susceptibility to in vivo degradation. ${ }^{4}$ Thus, out of over three thousand known AMPs, only seven of them have been approved and commercialized for use by the U.S. Food and Drug Administration. ${ }^{5}$ Notable examples are gramicidin D, which is used topically, and daptomycin, which is used intravenously. ${ }^{5}$

The antimicrobial mechanisms of many AMPs are often unclear, but three major models have been proposed: the carpet, toroidal pore, and barrel-stave models. ${ }^{6}$ The carpet model describes the cumulative action of AMPs interacting with the membrane surface that disrupts the bilayer through a detergent-like activity. In the barrel-stave and toroidal pore models, AMPs insert into the bilayer and oligomerize into pores that permeabilize the membrane. ${ }^{7}$

In all of these models, interactions with bacterial membranes are critical for activity, but it is challenging to study the polydisperse and dynamic interactions of AMPs with membranes. ${ }^{8}$ Native MS has been used to probe the influence of hydrophobic environments on peptide complex formation in nanodiscs, ${ }^{9}$ to investigate conformational preferences in vesicles, ${ }^{10}$ and to detect peptide variants in biological samples. ${ }^{11}$ We previously demonstrated that intact AMP-nanodisc complexes could be analyzed with native MS to determine the stoichiometry and specificity of incorporated AMPs in different bilayers. ${ }^{12,13,14}$ Nanodiscs are discoidal lipid bilayers used to solubilize membrane proteins and peptides in a native-like environment. However, despite recent advances in creating native MS- compatible nanodiscs that model natural lipid bilayers, ${ }^{15}$ native MS was previously unable to study interactions in nanodiscs made with natural lipid extracts due to the complexity of the bilayer. Additionally, native MS does not reveal whether the orientation of a peptide within the membrane is peripheral or integral.

To study AMP interactions in nanodiscs with natural lipid mixtures, we used fast photochemical oxidation of proteins (FPOP) in combination with LC-MS. FPOP is an irreversible footprinting technique that covalently labels solvent accessible residues on proteins. ${ }^{16}$ Hydroxyl radicals are generated in solution via photolysis, and these radicals then covalently modify solvent exposed amino acid side chains, resulting in predictable mass shifts that are detected via MS (Figure 1). Early hydroxyl radical footprinting experiments used a high energy synchrotron X-ray beam to generate radicals from water, ${ }^{17}$ but FPOP uses a $248 \mathrm{~nm} \mathrm{KrF}$ excimer laser to generate radicals from hydrogen peroxide. Recently, the use of a high-pressure flash lamp has emerged as a powerful platform for FPOP without the need for the laser.18 FPOP enables peptides to be directly probed in complex environments, and the fast timescale and relatively small size of the label ensures minimal disruptions and disturbances to the system ${ }^{19}$. Pioneering work has applied FPOP to membrane proteins in a variety of different environments ranging from detergent bicelles ${ }^{20}$ and micelles ${ }^{21}$ to nanodiscs 22 and picodiscs ${ }^{23}$ to whole organisms. ${ }^{24}$

Here, we employed FPOP to study AMP interactions in different nanodisc environments to study their lipid selectivity and provide insights into their mechanisms of toxicity. We compared oxidative labeling of two peptides, LL-37 and indolicidin, in the presence of three different lipid environments: phosphatidylcholine (PC), phosphatidylglycerol (PG), and E. coli lipid extracts. Additionally, we used native charge detection-mass spectrometry (CD-MS) to analyze nanodiscs produced from bacterial lipid extracts. We observed clear differences in peptide incorporation among each of the lipid environments. Indolicidin had the lowest levels of oxidation in DMPG nanodiscs, 
and LL-37 had the lowest levels of oxidation in E. coli polar lipid nanodiscs. Our results suggest that indolicidin interacts with lipid head groups via a simple, charge-driven mechanism, but that LL-37 interactions are more complex, preferring a diverse lipid environment for optimal antimicrobial activity.

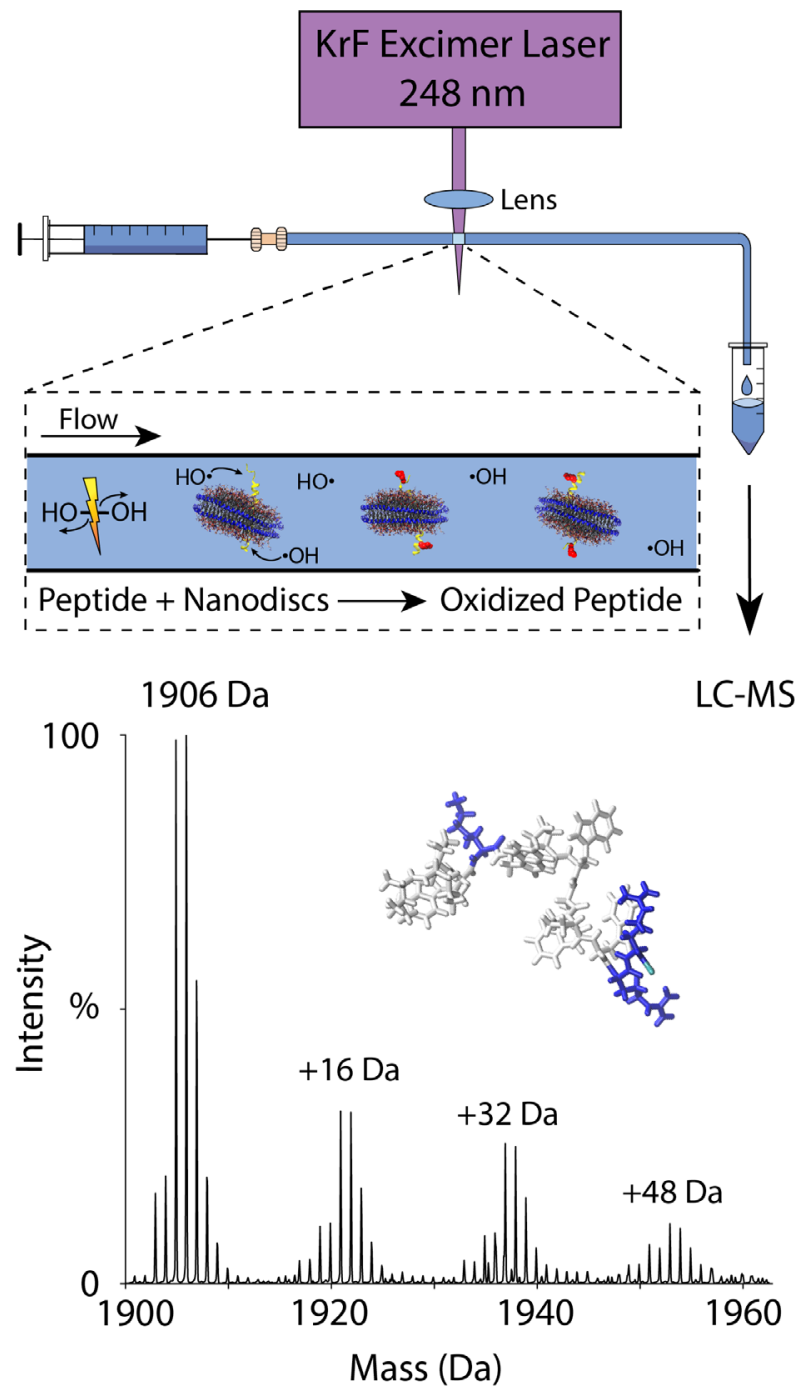

Figure 1. Schematic for FPOP (top) and an example deconvolved mass spectrum (bottom). Sample is flowed through a capillary and irradiated by a single pulse of $248 \mathrm{~nm}$ light, where photolysis of $\mathrm{H}_{2} \mathrm{O}_{2}$ produces hydroxyl radicals that label the solvent exposed residues of peptides in nanodiscs. Oxidation is then detected via MS, where each oxidation causes a +16 Da mass shift.

\section{EXPERIMENTAL SECTION}

Reagents. E. coli polar lipid extract, 1,2-dimyristoyl-sn-glycero-3-phosphocholine (DMPC), and 1,2-dimyristoyl-sn-glycero-3-phospho-(1'-rac-glycerol) (DMPG) lipids were purchased from Avanti Polar Lipids. Methionine, glutamine, phosphate buffered saline (PBS, bioperformance certified, $\mathrm{pH} 7.4$ ), catalase (from bovine liver), hydrogen peroxide $(\sim 30 \%)$, ammonium acetate, Amberlite XAD-2, and sodium cholate were purchased from Sigma-Aldrich. Methanol, isopropyl alcohol, and formic acid were purchased from Fischer. LL-37 was purchased from Bachem. Indolicidin was purchased from AnaSpec Inc. KrF gas was purchased from Linde Electronics and Specialty Gases.

Nanodisc assembly. Three types of nanodiscs were assembled: DMPG nanodiscs to model bacterial membranes, DMPC to model mammalian membranes, and $E$. coli lipid extract to reproduce bacterial membranes. Membrane scaffold protein MSP1D1(-) was expressed in E. coli and purified by immobilized metal affinity chromatography. This MSP was then used to make nanodiscs without incorporated peptide as previously described.25,26,27 Briefly, DMPC, DMPG, or E. coli lipids in chloroform were dried under nitrogen and solubilized in $0.1 \mathrm{M}$ sodium cholate. MSP1D1(-) was added to the lipids, and detergent was removed by addition of Amberlite XAD-2 hydrophobic beads with gentle agitation for at least 4 hours at room temperature. Nanodiscs were purified using size exclusion chromatography on a Superose 6 Increase 10/300 column (GE Healthcare) with $0.2 \mathrm{M}$ ammonium acetate at $\mathrm{pH} 6.8$.

FPOP oxidative labeling. The FPOP apparatus used a pulsed $248 \mathrm{~nm}$ KrF excimer laser (GAM Laser, EX350, Orlando, FL), a syringe pump, and a $150 \mu \mathrm{m}$ ID capillary. The capillary was prepared by burning off a small section of the protective coating, and then aligning the clear section so that it was in the direct path of the beam. Irradiation was performed at $5 \mathrm{~Hz}$ pulse frequency with a laser energy of at least $100 \mathrm{~mJ}$ per pulse as measured by an Ophir Photonics Juno pyroelectric energy meter.

Indolicidin stocks were prepared at $60 \mu \mathrm{M}, 180 \mu \mathrm{M}$, and 360 $\mu \mathrm{M}$ in 50:50 methanol: $\mathrm{H}_{2} \mathrm{O}$. LL-37 stocks were prepared at 60 $\mu \mathrm{M}$ and $180 \mu \mathrm{M}$ in 50:50 methanol: $\mathrm{H}_{2} \mathrm{O}$. Stock PBS was prepared at $0.1 \mathrm{M}$. Stock $\mathrm{H}_{2} \mathrm{O}_{2}$ was prepared at $\sim 100 \mathrm{mM}$ in PBS. Glutamine stock was prepared at $0.20 \mathrm{M}$ in PBS. Methionine stock was made at $0.19 \mathrm{M}$ in PBS. Catalase stock was prepared at $4.2 \mu \mathrm{M}$ in PBS. To keep the concentrations consistent with past studies using nanodiscs and AMPs, ${ }^{12,13}$ nanodisc stocks were made at $20 \mu \mathrm{M}$.

Peptides were added to the nanodiscs at 3:1, 9:1, and 18:1 peptide:nanodisc ratios in the FPOP solution at room temperature. Each ingredient was added separately in the order listed below for a final pre-FPOP solution volume of $50 \mu \mathrm{L}$.

Indolicidin samples at a 3:1 indolicidin:nanodisc ratio were prepared as follows: $30 \mu \mathrm{L}$ PBS (final concentration $80 \mathrm{mM}$ ), 5 $\mu \mathrm{L}$ glutamine (final $20 \mathrm{mM}$ ), $5 \mu \mathrm{L}$ nanodiscs (final $2.0 \mu \mathrm{M}$ ), $5 \mu \mathrm{L}$ $60 \mu \mathrm{M}$ indolicidin (final $6.0 \mu \mathrm{M}$ ), and $5 \mu \mathrm{L} \mathrm{H}_{2} \mathrm{O}_{2}$ (final $\sim 10 \mathrm{mM}$ ). 9:1 and 18:1 indolicidin:nanodisc samples were prepared similarly but with $5 \mu \mathrm{L}$ of either $180 \mu \mathrm{M}$ indolicidin (final $18.0 \mu \mathrm{M}$ ) or $360 \mu \mathrm{M}$ indolicidin (final $36.0 \mu \mathrm{M}$ ), respectively.

LL-37 samples at a 3:1 LL-37:nanodisc ratio were prepared as follows: $30 \mu \mathrm{L}$ PBS (final concentration $80 \mathrm{mM}$ ), $5 \mu \mathrm{L}$ glutamine (final $20 \mathrm{mM}$ ), $5 \mu \mathrm{L}$ nanodiscs (final $2.0 \mu \mathrm{M}$ ), $5 \mu \mathrm{L} 60 \mu \mathrm{M}$ LL-37 (final $6.0 \mu \mathrm{M}$ ), and $5 \mu \mathrm{L} \mathrm{H} \mathrm{H}_{2}$ (final $\sim 10 \mathrm{mM}$ ). 9:1 LL37:nanodiscs were prepared similarly but with $5 \mu \mathrm{L} 180 \mu \mathrm{M}$ LL37 (final $18.0 \mu \mathrm{M}$ ). 18:1 LL-37:nanodisc samples were prepared similarly but with $5 \mu \mathrm{L}$ of $10 \mu \mathrm{M}$ nanodiscs (final $1.0 \mu \mathrm{M}$ ) and $5 \mu \mathrm{L}$ of $180 \mu \mathrm{M}$ LL-37 (final $18.0 \mu \mathrm{M}$ ). The 18:1 LL-37 samples required a lower nanodisc concentration to account for the lower initial stock concentration of LL-37. To minimize preFPOP oxidation, $\mathrm{H}_{2} \mathrm{O}_{2}$ was added immediately prior to laser irradiation.

The sample was loaded into a Hamilton syringe, and a syringe pump was used to flow the solution through the FPOP system capillary. The flow rate was calculated to provide a $20 \%$ exclusion volume, ensuring no double exposure of the sample 
occurred. After laser exposure, the samples were collected immediately into vials containing $11 \mu \mathrm{L}$ of $0.19 \mathrm{M}$ methionine and $4.2 \mu \mathrm{M}$ catalase to quench the remaining hydroxyl radicals. The final sample volume after collection was $61 \mu \mathrm{L}$. Controls were produced in the same manner but without laser irradiation. At least three replicates per sample were performed for both controls and oxidized samples with separate nanodisc assemblies for each.

Liquid chromatography. After collection, separation was achieved using reversed phase liquid chromatography on an Infinity II HPLC (Agilent, Santa Clara, CA) equipped with a Waters Acquity UPLC C4 column $(1.7 \mu \mathrm{m}, 2.1 \times 100 \mathrm{~mm})$ held at $40^{\circ}$ C. Mobile phases were: A) $\mathrm{H}_{2} \mathrm{O}+0.1 \%$ formic acid and $\mathrm{B}$ ) $80 \%$ IPA in $\mathrm{H}_{2} \mathrm{O}+0.1 \%$ formic acid. A flow rate of $150 \mu \mathrm{L} / \mathrm{min}$ and a 32-minute gradient elution were performed as follows: $1 \% \mathrm{~B}$ from $0-7$ minutes, ramp $1 \% \mathrm{~B}$ to $99 \% \mathrm{~B}$ from $7-12$ minutes, hold $99 \%$ B from $12-17$ minutes, ramp $99 \%$ B to $1 \%$ B from 17-24 minutes, and then hold 1\% B from 24-32 minutes. Under this separation scheme, peptides had an elution time of around 16 minutes.

Although a C18 column is typically used for peptide separations, we used a C4 column to simplify data analysis and allow both labeled and non-labeled peptides to elute simultaneously. This simultaneous elution enables precise quantitation of the oxidation ratio because the oxidized and unoxidized peptides are eluting at the same point in the gradient. Due to the high lipid content of the samples, the C4 column also allowed for easier elution of the lipids.

Mass spectrometry and data analysis. Mass analysis was performed on a Synapt XS time-of-flight mass spectrometer (Waters Corporation, Milford, MA). ESI-MS in positive mode was performed on the LC eluent throughout the entire 32-minute separation for each sample. Relevant parameters are as follows: capillary voltage $1.3 \mathrm{kV}$, sample cone voltage $30 \mathrm{~V}$, source temperature $30^{\circ} \mathrm{C}$, cone gas $100 \mathrm{~L} / \mathrm{hr}$, desolvation gas $700 \mathrm{~L} / \mathrm{hr}$, nebulizer gas pressure $6.5 \mathrm{bar}$, extended dynamic range, and sensitivity mode ( $\mathrm{V}$ mode) without ion mobility. Data analysis was performed using UniDec deconvolution software. ${ }^{28}$ Briefly, the peptide peak in the chromatogram was selected, the $m / z$ spectrum was deconvolved in UniDec, oxidized peaks were identified, and the percentage of oxidation as determined by the first oxidation peak was calculated. Specifically, the percent oxidation is the quotient of the highest intensity of the first oxidation peak divided by the highest intensity of the non-oxidized peak, multiplied by 100 . Student's t-tests at the $95 \%$ confidence interval were used for statistical analyses.

Charge detection-mass spectrometry. CD-MS was performed on a Q-Exactive HF equipped with ultra-high mass range modifications (Thermo Fisher Scientific, Bremen). MS parameters used for analyzing $E$. coli nanodiscs have been described previously. ${ }^{26}$ Briefly, the resolution was set to 240,000 , and the trapping gas pressure was set to 1 , which corresponds to an ultrahigh vacuum pressure in the Orbitrap of $6.40 \times 10^{-11}$ $8.12 \times 10^{-11}$ mbar. The noise threshold was set to 0 . CD-MS data sets contained at least 5,000 ions and were collected for 5-10 minutes. For $E$. coli nanodiscs with and without peptide, the higher-energy C-trap dissociation (HCD) voltage was set to 0 $20 \mathrm{~V}$ to increase the number of higher mass ions. CD-MS datasets were then deconvolved with UniDecCD (UCD), which lowered the charge uncertainty and increased the resolution of E. coli nanodiscs. UCD is part of the UniDec open-source software package ${ }^{28}$ and the deconvolution algorithm has been previously described. ${ }^{26}$ Measurements were collected on three different nanodisc assemblies, and results are reported as the average and standard deviation of the three replicates.

\section{RESULTS AND DISCUSSION}

FPOP of indolicidin. Due to the cationic nature of these AMPs, we predicted that both LL-37 and indolicidin would have the highest affinity for highly anionic DMPG nanodiscs, the lowest affinity for zwitterionic DMPC nanodiscs, and an intermediate affinity for $E$. coli nanodiscs, which contain roughly two thirds neutral PE lipids and one third anionic cardiolipin and PG lipids. ${ }^{29}$ Higher affinity for lipids should result in more protection of the membrane-embedded peptide and thus lower oxidation.
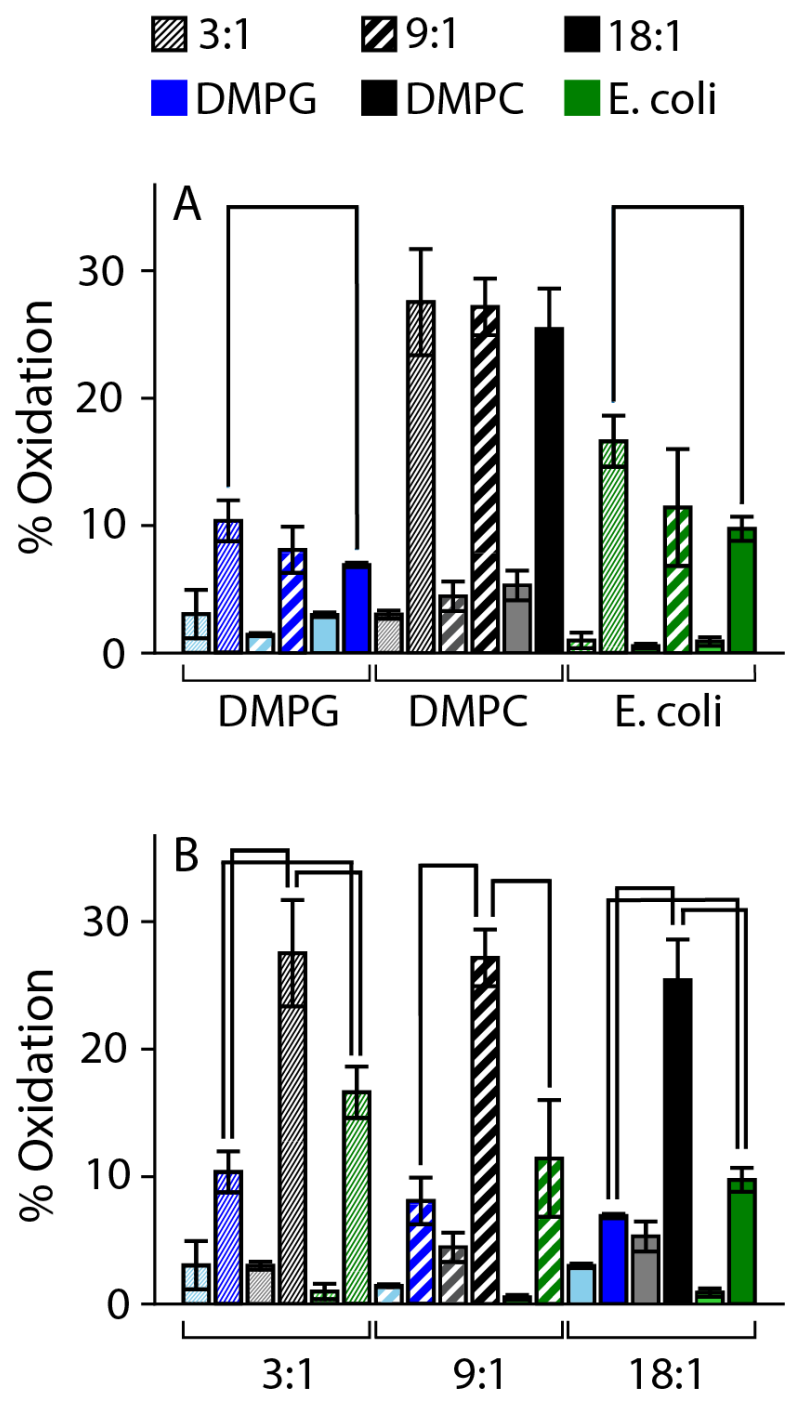

Figure 2. Indolicidin oxidation grouped by peptide:nanodisc ratio (A) and by lipid (B). Each lipid is indicated by color, with DMPG in blue, DMPC in black, and E. coli in green. Peptide:nanodisc ratios are represented by different shading, with solid shading representing 18:1, thick shading indicating 9:1, and thin shading indicating 3:1. The left bar in each pair is the corresponding control with no UV laser irradiation. Statistically significant differences in oxidation are indicated by black lines connecting the bars. 
Indolicidin behaved predictably in each of the nanodisc variants we tested. The lowest levels of oxidation were observed in DMPG nanodiscs (Figure 2), indicating that indolicidin is more protected and thus more incorporated in DMPG bilayers. The highest levels of oxidation were in DMPC nanodiscs, indicating that indolicidin was less protected and thus less incorporated in DMPC bilayers. Oxidation levels of indolicidin in $E$. coli nanodiscs fell in between those of the PG and PC nanodiscs. Overall, these data are consistent with indolicidin-membrane interactions being driven primarily by the degree of anionic lipid head groups present in the membrane.
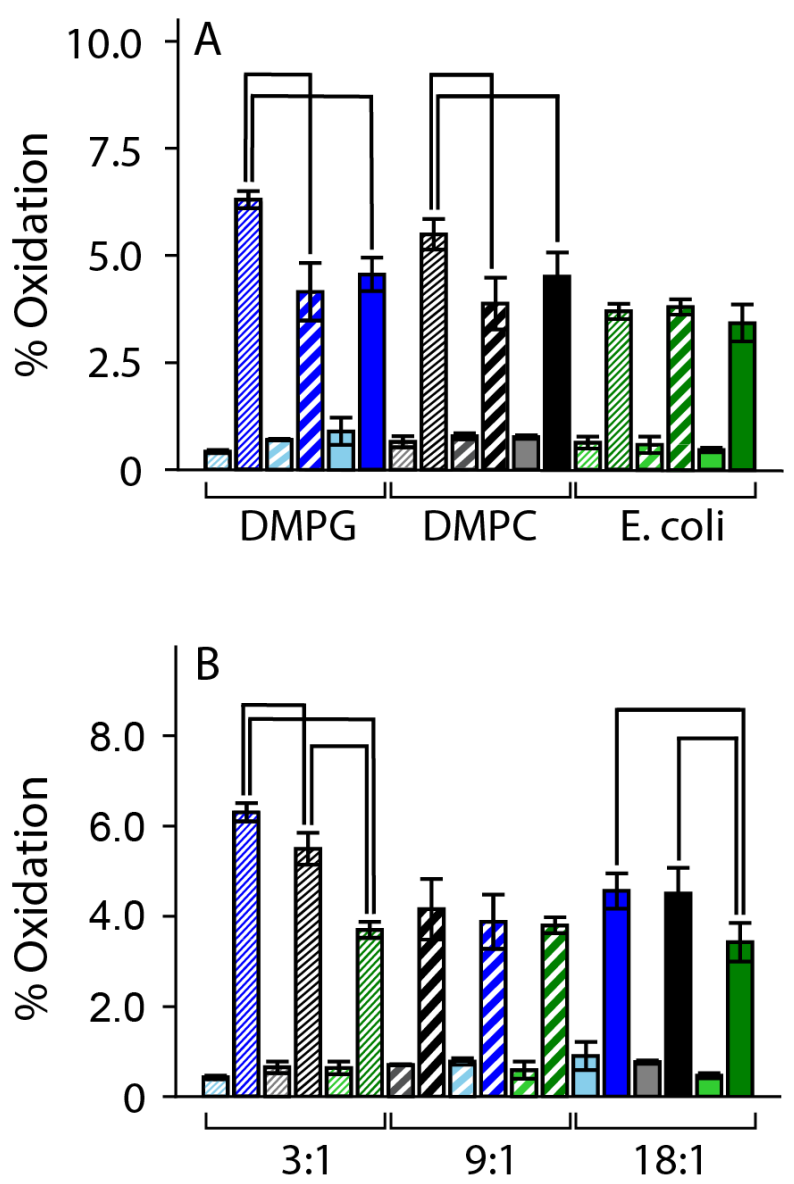

Figure 3. LL-37 oxidation grouped by lipid (A) and by peptide:nanodisc ratio (B). Each lipid is indicated by color, with DMPG in blue, DMPC in black, and E. coli in green. Peptide:nanodisc ratios are represented by different shading, with solid shading representing 18:1, thick shading indicating 9:1, and thin shading indicating 3:1. The left bar in each pair is the corresponding control with no UV laser irradiation. Statistically significant differences in oxidation are indicated by black lines connecting the bars.

Next, we investigated the effects of indolicidin concentration by altering the peptide:nanodisc ratios. A comparison of indolicidin oxidation at $3: 1,9: 1$, and 18:1 peptide:nanodisc ratios generally revealed slightly increased protection at higher ratios (Figure 2), suggesting higher indolicidin incorporation at higher ratios. Overall, these data agree with previous results on

indolicidin incorporation into nanodiscs, which indicated that it had a strong preference for DMPG lipids, increasing incorporation at higher concentrations, but minimal selectivity for specific oligomeric states. ${ }^{13}$ Thus, indolicidin seems to have simple charge-driven interactions with anionic lipid head groups.

FPOP of LL-37. Because LL-37 is also cationic, we predicted it would also be more protected in DMPG nanodiscs and less protected in DMPC nanodiscs. However, except for a slight difference at 3:1 peptide:nanodisc, no significant differences in LL-37 oxidation were observed between DMPC and DMPG nanodiscs (Figure 3). Looking at concentration effects, DMPG and DMPC had higher levels of oxidation of LL-37 at a 3:1 ratio. These results suggest that at lower peptide:nanodisc ratios, LL37 is less buried in the membrane, resulting in greater solvent exposure and decreased protection from oxidation. Comparable levels of oxidation were observed in both DMPG and DMPC nanodiscs at 9:1 and 18:1 ratios. One explanation for this behavior is that the affinity of LL-37 for both DMPC and DMPG nanodiscs may be essentially equal, despite likely having different methods of interaction. LL-37 destabilizes DMPC nanodiscs above a $6: 1$ ratio, ${ }^{12}$ but these data suggest that it is likely still associated with lipid aggregates even though it is no longer embedded within intact nanodiscs. In DMPG nanodiscs, a similar plateau of oxidation is observed above 3:1. Comparison with prior native MS data ${ }^{12,13}$ indicates that this plateau is likely due to formation of increasingly larger oligomeric complexes in the membrane that are protected.

Unlike indolicidin, the lowest levels of oxidation for LL-37 were observed with $E$. coli extract nanodiscs, indicating that LL-37 incorporated the most into bilayers with these natural lipids. We previously found a similar increase in incorporation for LL-37 in simple model bacterial membrane nanodiscs composed of PG, phosphatidylethanolamine, and cardiolipin, ${ }^{15}$ indicating that LL-37 may have evolved to target membranes with more complex lipid composition. Interestingly, unlike DMPC and DMPG, oxidation levels remained unchanged across all three ratios (Figure 3), indicating a high affinity even at low ratios of peptide:nanodisc.

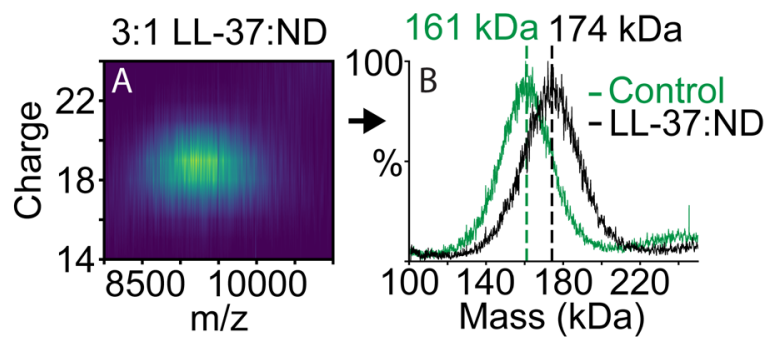

Figure 4. CD-MS of E. coli nanodiscs with LL-37. A) Deconvolved charge vs $\mathrm{m} / \mathrm{z}$ histogram from CD-MS of E. coli nanodiscs at a 3:1 LL-37:nanodisc ratio. B) Overlayed deconvolved mass distributions of E. coli control nanodiscs in green and a 3:1 LL-37:nanodisc ratio in black. The mass shift from the center of the mass distribution of the control, indicated by the dashed lines, shows an increase of $13.7 \pm 7.1 \mathrm{kDa}$, which corresponds to $3 \pm 1.6 \mathrm{LL}-37$ peptides.

CD-MS of LL-37 in E. coli nanodiscs. Unfortunately, it is not possible to perform conventional native MS on $E$. coli nanodiscs due to the complexity of the mass distribution. To address this challenge, we used single-ion CD-MS to measure the broad mass distributions of $E$. coli nanodiscs with LL-37. At a 3:1 ratio 
of LL-37:nanodisc, the center of mass shifted by $13.7 \pm 7.1 \mathrm{kDa}$, which corresponds to an addition of $3 \pm 1.6$ LL-37 peptides (Figure 4). Interestingly, at 9:1 and 18:1 ratios, E. coli nanodiscs are destroyed, with the majority of signal coming from 2 free MSP belts. However, the consistently low oxidation at these higher ratios (Figure 3) suggests that LL-37 is still surrounded by lipids and may be forming lipid-peptide oligomers that follow more of a carpet mechanism. The combination of the FPOP and CD-MS data suggests that LL-37 initially incorporates into $E$. coli nanodiscs with high affinity but may proceed to act with a carpet mechanism at higher ratios.

\section{CONCLUSION}

Here, we demonstrated that FPOP of AMPs in nanodiscs provides unique complementary information on peptide-lipid interactions within a variety of biologically-relevant lipid environments. This method provides a reliable way to investigate interactions that are difficult to study with other techniques and has the potential to provide insights into the extent of AMP insertion into bilayers. We found that indolicidin likely participates in charge driven interactions with the surrounding lipid environment, where the cationic charge is attracted to the anionic lipids.

Although LL-37 is overall cationic, we observed similar levels of protection in both DMPG and DMPC nanodiscs, and the highest protection was in E. coli lipid nanodiscs. Therefore, LL37 interacts with both anionic and zwitterionic bilayers, but optimal peptide-lipid interactions are achieved when the membrane contains a mixture of anionic and zwitterionic lipids. Protection from oxidation is also likely influenced by the tail lengths and fluidity of the lipids in $E$. coli extract nanodiscs. ${ }^{30}$ These data indicate that, unlike indolicidin, the specificity of LL-37 does not proceed via a simple charge driven mechanism. Instead, LL-37 may be specifically evolved to interact with and disrupt bacterial membranes.

Future research will expand this approach to use MS/MS analysis to provide residue level information on solvent exposed regions of labeled peptides, which will give higher resolution information regarding AMP structure and mechanisms. Overall, FPOP of AMPs in nanodiscs allows the elucidation of complex AMP-lipid interactions and-when coupled with CDMS, conventional native MS, or both-reveals insights to their mechanism of action.

\section{AUTHOR INFORMATION}

\section{Corresponding Author}

*mtmarty@arizona.edu

\section{Notes}

The authors declare no competing financial interests.

\section{ACKNOWLEDGMENT}

The authors thank Lisa Jones and Michael Gross for helpful suggestions on setting up the FPOP system. They also thank Maria Reinhardt-Szyba, Kyle Fort, and Alexander Makarov at Thermo Fisher Scientific for their support on the UHMR Q-Exactive HF instrument. The pMSP1D1 plasmid was a gift from Stephen Sligar (Addgene plasmid \#20061). This work was funded by the National Institutes of Health (R35 GM128624). The content is solely the responsibility of the authors and does not necessarily represent the official views of the National Institutes of Health

\section{REFERENCES}

(1) Boto, A.; De La Lastra, J. M. P.; González, C. C. The Road from Host-Defense Peptides to a New Generation of Antimicrobial Drugs. Molecules 2018, 23 (2).

(2) Peschel, A.; Sahl, H. G. The Co-Evolution of Host Cationic Antimicrobial Peptides and Microbial Resistance. Nat. Rev. Microbiol. 2006, 4 (7), 529-536.

(3) Joo, H. S.; Fu, C. I.; Otto, M. Bacterial Strategies of Resistance to Antimicrobial Peptides. Philos. Trans. R. Soc. B Biol. Sci. 2016, 371 (1695)

(4) Thapa, R. K.; Diep, D. B.; Tønnesen, H. H. Topical Antimicrobial Peptide Formulations for Wound Healing: Current Developments and Future Prospects. Acta Biomater. 2020, 103, 52-67.

(5) Chen, C. H.; Lu, T. K. Development and Challenges of Antimicrobial Peptides for Therapeutic Applications. Antibiotics 2020, 9 (1)

(6) Park, Y. K.; Hahm, K. S. Antimicrobial peptides (AMPs): peptide structure and mode of action. BMB Rep. 2005. 38 (5), 507-516.

(7) Wimley, W. C. Describing the mechanism of antimicrobial peptide action with the interfacial activity model. ACS Chem. Biol. 2010, 5 (10), 905-917.

(8) Liu, X. R.; Zhang, M. M.; Gross, M. L. Mass Spectrometry-Based Protein Footprinting for Higher-Order Structure Analysis: Fundamentals and Applications. Chem. Rev. 2020, 120 (10), $4355-4454$.

(9) Li, J.; Richards, M. R.; Kitova, E. N.; Klassen, J. S. Delivering Transmembrane Peptide Complexes to the Gas Phase Using Nanodiscs and Electrospray Ionization. J. Am. Soc. Mass Spectrom. 2017, 28 (10), 2054-2065.

(10) Patrick, J. W.; Gamez, R. C.; Russell, D. H. The Influence of Lipid Bilayer Physicochemical Properties on Gramicidin A Conformer Preferences. Biophys. J. 2016, 110 (8), 1826-1835.

(11) Al-Adwani, S.; Wallin, C.; Balhuizen, M. D.; Veldhuizen, E. J. A.; Coorens, M.; Landreh, M.; Végvári, Á.; Smith, M. E.; Qvarfordt, I.; Lindén, A.; et al. Studies on Citrullinated LL-37: Detection in Human Airways, Antibacterial Effects and Biophysical Properties. Sci. Rep. 2020, 10 (1), 1-14.

(12) Walker, L. R.; Marzluff, E. M.; Townsend, J. A.; Resager, W. C.; Marty, M. T. Native Mass Spectrometry of Antimicrobial Peptides in Lipid Nanodiscs Elucidates Complex Assembly. Anal. Chem. 2019, 91 (14), 9284-9291.

(13) Walker, L. R.; Marty, M. T. Revealing the Specificity of a Range of Antimicrobial Peptides in Lipid Nanodiscs by Native Mass Spectrometry. Biochemistry 2020, 59 (23), 2135-2142.

(14) Walker, L. R.; Marty, M. T. Lipid Tails Modulate Antimicrobial Peptide Membrane Incorporation and Activity. bioRxiv 2021, 2021.08.12.456135.

(15) Kostelic, M. M.; Zak, C. K.; Jayasekera, H. S.; Marty, M. T. Assembly of Model Membrane Nanodiscs for Native Mass Spectrometry. Anal. Chem. 2021, 93 (14), 5972-5979.

Hambly, D. M.; Gross, M. L. Laser Flash Photolysis of Hydrogen Peroxide to Oxidize Protein Solvent-Accessible Residues on the Microsecond Timescale. J. Am. Soc. Mass Spectrom. 2005, 16 (12), 2057-2063.

Maleknia, S. D.; Brenowitz, M.; Chance, M. R. Millisecond Radiolytic Modification of Peptides by Synchrotron X-Rays Identified by Mass Spectrometry. Anal. Chem. 1999, 71 (18), 3965-3973.

Sharp, J. S.; Chea, E. E.; Misra, S. K.; Orlando, R.; Popov, M.; Egan, R. W.; Holman, D.; Weinberger, S. R. Flash Oxidation (FOX) System: A Novel Laser-Free Fast Photochemical Oxidation Protein Footprinting Platform. J. Am. Soc. Mass Spectrom. 2021, 32 (7), 1601-1609.

(19) Gau, B. C.; Sharp, J. S.; Rempel, D. L.; Gross, M. L. Fast Photochemical Oxidation of Protein Footprints Faster than Protein Unfolding. Anal. Chem. 2009, 81 (16), 6563-6571.

(20) Pan, Y.; Brown, L.; Konermann, L. Kinetic Folding Mechanism of an Integral Membrane Protein Examined by Pulsed Oxidative Labeling and Mass Spectrometry. J. Mol. Biol. 2011, 410 (1), 146-158.

Pan, Y.; Ruan, X.; Valvano, M. A.; Konermann, L. Validation of 
Membrane Protein Topology Models by Oxidative Labeling and Mass Spectrometry. J. Am. Soc. Mass Spectrom. 2012, 23 (5), 889-898.

(22) Lu, Y.; Zhang, H.; Niedzwiedzki, D. M.; Jiang, J.; Blankenship, R. E.; Gross, M. L. Fast Photochemical Oxidation of Proteins Maps the Topology of Intrinsic Membrane Proteins: LightHarvesting Complex 2 in a Nanodisc. Anal. Chem. 2016, 88 (17), 8827-8834

(23) Zhou, F.; Yang, Y.; Chemuru, S.; Cui, W.; Liu, S.; Gross, M.; Li, W. Footprinting Mass Spectrometry of Membrane Proteins: Ferroportin Reconstituted in Saposin A Picodiscs. Anal. Chem. 2021.

(24) Espino, J. A.; Zhang, Z;; Jones, L. M. Chemical Penetration Enhancers Increase Hydrogen Peroxide Uptake in C. Elegans for in Vivo Fast Photochemical 0xidation of Proteins. J. Proteome Res. 2020, 19 (9), 3708-3715.

(25) Reid, D. J.; Keener, J. E.; Wheeler, A. P.; Zambrano, D. E. Diesing, J. M.; Reinhardt-Szyba, M.; Makarov, A.; Marty, M. T. Engineering Nanodisc Scaffold Proteins for Native Mass Spectrometry. Anal. Chem. 2017, 89 (21), 11189-11192.

(26) Kostelic, M.; Zak, C.; Liu, Y.; Chen, V.; Wu, Z.; Sivinski, J.;
Chapman, E.; Marty, M. UniDecCD: Deconvolution of Charge Detection-Mass Spectrometry Data. ChemRxiv, 2021. DOI: 10.33774/chemrxiv-2021-d2d6z DOI: 10.33774/chemrxiv2021-d2d6z.

(27) Denisov, I. G.; Grinkova, Y. V.; Lazarides, A. A.; Sligar, S. G. Directed Self-Assembly of Monodisperse Phospholipid Bilayer Nanodiscs with Controlled Size. J. Am. Chem. Soc. 2004, 126 (11), 3477-3487.

(28) Marty, M. T.; Baldwin, A. J.; Marklund, E. G.; Hochberg, G. K. A. Benesch, J. L. P.; Robinson, C. V. Bayesian Deconvolution of Mass and Ion Mobility Spectra: From Binary Interactions to Polydisperse Ensembles. Anal. Chem. 2015, 87 (8), 43704376.

(29) Avanti Polar Lipids Product Page. https://avantilipids.com/product/100600 (accessed Oct 8, 2021).

(30) Walker, L. R.; Marty, M. T. Lipid Tails Modulate Antimicrobial Peptide Membrane Incorporation and Activity. bioRxiv, 2021 DOI: https://doi.org/10.1101/2021.08.12.456135.

\section{For Table of Contents:}

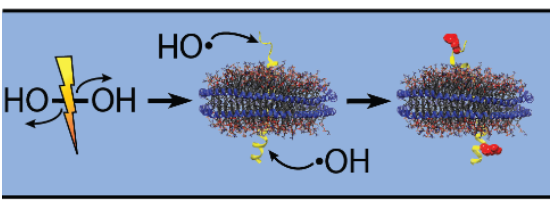

